\title{
EFFECTS OF PROBIOTICS ON LIPID PROFILES IN HYPERCHOLESTEROLEMIA ADULT PATIENTS: AN EVIDENCE-BASED CASE REPORT
}

\author{
Elisa $^{1}$, Wiji Lestari ${ }^{2}$ \\ ${ }^{1}$ Department of Nutrition, Faculty of Medicine Universitas Indonesia - Dr. Cipto Mangunkusumo Hospital, \\ Jakarta, Indonesia \\ ${ }^{2}$ Department of Nutrition, Faculty of Medicine Universitas Indonesia - Dr. Cipto Mangunkusumo Hospital, \\ Jakarta, Indonesia \\ *Corresponding author, contact: wijilestari.doc@gmail.com
}

\begin{abstract}
Background : Hypercholesterolemia prevalence was $39 \%$ in adults and a risk factor for cardiovascular, cerebrovascular, atherosclerotic disease, leading to vertigo. Diet and specific food potentiate in dyslipidemia risk reduction. Several studies have been proposed that probiotics have a hypocholesterolemic effect.

Objectives : This study aims to determine the effect of probiotics on lipid profiles in hypercholesterolemia adult patients.

Methods : Electronic literature searching was conducted from Pubmed, Scopus, and Cochrane Library. MeSH Terms combined with Title/Abstract are used in advanced search based on inclusion and exclusion criteria.

Results : Critical appraisal was conducted for a systematic review and meta-analysis of randomized controlled trials. Probiotic supplementation significantly decreases total cholesterol and low-density lipoprotein cholesterol levels but not statistically significant in high-density lipoprotein and triglycerides levels.

Conclusion : Single strain Lactobacillus plantarum probiotics for at least six weeks can improve total cholesterol levels in hypercholesterolemia adult patients.
\end{abstract}

Keywords : probiotics, hypercholesterolemia, lipid profile

\section{Clinical Scenario}

A 45-year-old man was admitted to the hospital with a chief complaint of dizziness. His dizziness got worse with a change of position. He also complains of nausea and vomit. He had a history of high lipid profiles, but he did not take any lower lipid agent regularly. His blood pressure was $120 / 78 \mathrm{mmHg}$ from the physical examination, the other vital signs were within normal limit, and nystagmus positive. His laboratory results were hypercholesterolemia with total cholesterol (TC) level $227 \mathrm{mg} / \mathrm{dL}$, low-density lipoprotein cholesterol (LDL-C) $126 \mathrm{mg} / \mathrm{dL}$, high-density lipoprotein cholesterol (HDL-C) $62 \mathrm{mg} / \mathrm{dL}$, and triglycerides (TG) $46 \mathrm{mg} / \mathrm{dL}$. The neurologist prescribed him simvastatin $10 \mathrm{mg}$ once daily, but he refused to consume it because of fear that statins might lower his libido. Then, he also was referred to the clinical nutrition specialist to control his cholesterol level and asked about food supplementation, which could be lowering his cholesterol level. The 
clinical nutrition specialist considered giving him probiotics supplementation to improve his lipid profiles.

\section{Introduction}

In 2008, World Health Organization (WHO) reported elevated blood cholesterol level or hypercholesterolemia prevalence was $39 \%$ worldwide in adults 25 years old and older. ${ }^{1}$ Causes of hypercholesterolemia are primary and secondary. The primary causes are a familial or genetic mutation of the LDL receptor gene, defective apolipoprotein

B, polygenic hypercholesterolemia, and proprotein convertase subtilisin/ kexin type 9 gene gainof-function mutation. The secondary causes are medical conditions such as diabetes mellitus, hypothyroidism, cholestasis, and nephrotic syndrome. Excessive dietary intake of cholesterol, smoking, and medications such as thiazide diuretics and cyclosporine are linked to increased hypercholesterolemia. $^{2}$

Hypercholesterolemia is a risk factor for cardiovascular, cerebrovascular, atherosclerotic disease, ${ }^{3}$ including vestibulocochlear vessels that may lead to vertigo. It is a treatable cause of vestibular vertigo. ${ }^{4}$ Diet has a crucial role in control non-communicable diseases. Specific food has a potential reduction in the risk of dyslipidemia. ${ }^{5}$ Intestinal microbiota is responsible for many biochemical reactions and regulates metabolic status. Hypercholesterolemia persons have lower bacterial diversity in the intestinal compared with controls. Dysbiosis can directly emerge and result in non-communicable disease, which presents with dyslipidemia. Several studies had been investigated the hypocholesterolemic effects of probiotics, and have different results. ${ }^{6}$ Therefore, we conduct evidence-based literature searching if probiotics have positive results in improving lipid profile in hypercholesterolemia adult patients or not.

\section{Clinical Question}

The clinical question of this evidence-based case report is: Can probiotics help improve lipid profile in hypercholesterolemia patients?

Population (P): Adult patient with hypercholesterolemia

Intervention (I): Probiotic supplementation Comparison (C): No intervention or placebo Outcome (O): Lipid profile level

\section{Methods}

\section{Search Strategy}

Literature searching used advanced search from Pubmed, Scopus, and Cochrane Library on June 1st, 2021. Keywords used in Medical Subject Headings (MeSH) Terms and

Title/Abstract

are 
"hypercholesterolemia", hypercholesterol*”, "probiotic", "probiotics", and "lipid profil*". We used the asterisk $(*)$ to broaden search by finding words start with the same letters. The results were screened for duplication using the EndNote application. After duplication filtered, literature was matched for title and abstract which suitable with PICO. Literature in full text and appropriate with PICO will be appraised after met the eligibility criteria.

\section{Eligibility Criteria}

Article selection was made based on inclusion and exclusion criteria which in line with the clinical question. The inclusion criteria were: 1) adult patients with hypercholesterolemia; 2) patients who receive probiotic supplementation intervention; 3) study design in the systematic review, meta-analysis, or randomized controlled trial; 4) lipid profile level as the outcome; 5) publication within the last five years. The exclusion criteria were: 1) not available in full text;2) studies not in human; 3) not in the English language.

\section{Results}

There were 151 articles found in Pubmed, five articles in Cochrane Library, and 69 articles in Scopus (Table 1). After removing duplication and was screened based on inclusion and exclusion criteria, there were six articles. One article was limited access, and one article was included in the systematic review. After double filtering, two articles were excluded because they had no specified hypercholesterolemia adult, and one article had no suitable comparison with a clinical question. Hence, the study included for appraisal was one article (Figure 1). The article had same study characteristics (Table 2) and relevance with our clinical question (Table 3), level of evidence 1 and was a systematic review and meta-analysis of randomized controlled trials (Table 4).

\section{Critical Appraisal}

Critical appraisal was done by using the method for systematic review (Table 5) for finding, appraisal, include, total up, and heterogeneity of PICO (FAITH) from the Center of Evidence-Based Medicine (CEBM).

\section{Discussion}

Several studies have been proposed that probiotics have a hypocholesterolemic effect by binding cholesterol in the small intestine. Probiotics microorganisms can break down bile salt by synthesizing bile salt hydrolase (BSH) enzyme. ${ }^{7}$ Lactic acid bacteria (LAB) such as Lactobacilli and Bifidobacteria have BSH activity. ${ }^{8}$ The enzyme deconjugates bile salt in the enterohepatic circulation by hydrolyzing 
conjugated glycodeoxycholic acid and taurodeoxycholic acid to free taurine, glycine, and free cholic acid. Free taurine and glycine will be re-entered to the liver, and free cholic acid will be excreted into feces. ${ }^{7}$ Deconjugated bile salt is less soluble and less reabsorbed in the intestine so that it will be more free bile acids excretion in feces and less soluble and absorption lipid in the gut. Cholesterol is the precursor of bile acids. It will be converted to bile acid to replace its loss during excretion, leading to reduced serum cholesterol level. ${ }^{8}$

In the meta-analysis conducted by Mo R, et al. ${ }^{9}$ included 19 randomized controlled trials of hypercholesterolemia patient with baseline total cholesterol level > $200 \mathrm{mg} / \mathrm{dL}$ or $5.18 \mathrm{mmol} / \mathrm{L}$, who did not receive any cholesterol-lowering agents. The probiotics effects on lipid profiles had different outcome in TC, LDL-C, HDL-C, and TG level. Total cholesterol level changes $-0.25 \mathrm{mmol} / \mathrm{L}(95 \% \mathrm{CI}:-0.39--0.12 ; \mathrm{p}=$ $0.0002)$ with heterogeneity $\left(I^{2}=85 \%, \mathrm{p}<\right.$ $0.01)$, LDL-C level changes $-0.17 \mathrm{mmol} / \mathrm{L}$ (95\% CI: $-0.25--0.09 ; \mathrm{p}<0.0001)$ with heterogeneity $\left(I^{2}=67 \%, \mathrm{p}<0.01\right)$, HDL-C level changes $-0.02 \mathrm{mmol} / \mathrm{L}$ (95\% CI: -0.05 $0.02 ; \mathrm{p}=0.37)$ with heterogeneity $\left(I^{2}=80 \%\right.$, $\mathrm{p}<0.01$ ), and TG level changes $0.01 \mathrm{mmol} / \mathrm{L}$ (95\% CI: $-0.02-0.05 ; \mathrm{p}=0.51)$ without heterogeneity $\left(I^{2}=0 \%, \mathrm{p}=0.71\right)$. The conversion factors are $1 \mathrm{mg} / \mathrm{dL}=0.0259$ $\mathrm{mmol} / \mathrm{L}$ for TC, LDL-C and HDL-C, and 1 $\mathrm{mg} / \mathrm{dL}=0.0113 \mathrm{mmol} / \mathrm{L}$ for TG. From these data results mean that probiotic supplementation can significantly decreased TC and LDL-C level, but not statistically significant in HDL-C and TG level.

Subgroup analysis showed that probiotics had more significant effects on reduced TC and LDL-C levels in mild hypercholesterolemia subjects (TC 5.18$6.22 \mathrm{mmol} / \mathrm{L})$ than in moderate hypercholesterolemia (TC > $6.22 \mathrm{mmol} / \mathrm{L}$ ), younger than 50 years old, intervention duration for six weeks or more. Single strain probiotics had a more significant reduction in TC level than multi-strain but had the opposite result in reducing LDL-C. In lowering TC and LDL-C levels, the Lactobacillus plantarum strain had a significant effect. The use of fermented milk products had reduced LDL-C level more significant, but there was no difference between probiotic forms in reducing TC. The Lactobacillus plantarum dose used in the original trials were $2 \times 10^{9} \mathrm{CFU} /$ capsule (twice daily), $1.2 \times 10^{9} \mathrm{CFU}$ daily, 1.28-3.01 x $10^{9} \mathrm{CFU}$ daily, and $5 \times 10^{7} \mathrm{CFU} / \mathrm{mL}$ (400 $\mathrm{mL}$ daily in a rose-hip drink). ${ }^{9}$

Probiotics in the small intestine can adsorb cholesterol to lower free cholesterol levels to absorb in the intestine., 
Lactobacillus strain may produce cholesterol reductase in catalyzing the conversion of cholesterol to coprostanol in the intracellular and extracellular, ${ }^{10}$ ferulic acid which inhibits 3-hydroxy-3-methylglutaryl coenzyme A (HMG-CoA) reductase enzyme which has a role in endogenous cholesterol synthesis. ${ }^{11}$ Higher short-chain fatty acids (SCFA) levels produced by probiotics could lower cholesterol levels by blocking hepatic cholesterol synthesis. ${ }^{8}$

This study's limitations include studies with significant heterogeneity and could not conclude the reason for this heterogeneity; selection bias may occur because excluded studies with insufficient information; and restricted English language may cause publication bias. In addition, the strength of this study is specified in the hypercholesterolemia adult population and conducted subgroup analyses. ${ }^{9}$ We suggest that larger randomized controlled trials with many homogenous samples and studies focused on how much defined strain probiotics dose to make sure the effects of the probiotics on lipid profile.

\section{Conclusion}

In this case, a 45-year-old hypercholesterolemia man can be supplemented with single-strain Lactobacillus plantarum probiotics for at least six weeks to improve total cholesterol levels.

Hence, improving hypercholesterolemia outcomes can be combined with cholesterol-lowering drugs, lifestyle modification, a low cholesterol diet, and Lactobacillus plantarum probiotics supplementation in adequate amounts.

\section{Competing Interest}

Authors declared no conflict of interest in this study.

\section{List of Abbreviations}

\begin{tabular}{|c|c|}
\hline BSH & : bile salt hydrolase \\
\hline $\mathrm{C}$ & : comparison \\
\hline CEBM & $\begin{array}{l}\text { : Center of Evidence-Based } \\
\text { Medicine }\end{array}$ \\
\hline FAITH & $\begin{array}{l}\text { : finding, appraisal, include, } \\
\text { total up, and heterogeneity }\end{array}$ \\
\hline HDL-C & $\begin{array}{l}\text { : high-density lipoprotein } \\
\text { cholesterol }\end{array}$ \\
\hline HMG-CoA & $\begin{array}{l}\text { : 3-hydroxy-3-methylglutaryl } \\
\text { coenzyme A }\end{array}$ \\
\hline I & : intervention \\
\hline LAB & : lactic acid bacteria \\
\hline LCL-C & $\begin{array}{l}\text { : low-density } \\
\text { cholesterol }\end{array}$ \\
\hline $\mathrm{MeSH}$ & : Medical Subject Headings \\
\hline $\mathrm{O}$ & : outcome \\
\hline $\mathrm{P}$ & : population \\
\hline SCFA & : short-chain fatty acids \\
\hline $\mathrm{TC}$ & : total cholesterol \\
\hline TG & : triglycerides \\
\hline WHO & : World Health Organization \\
\hline
\end{tabular}

\section{References}

1. WHO. Raised total cholesterol $(\geq 5.0$ $\mathrm{mmol} / \mathrm{L}$ ) data by WHO region [cited 2021 June 15]. Available from: https://apps.who.int/gho/data/view.main.25 70?lang=en.

2. Al-Zahrani J, Shubair MM, Al-Ghamdi S, Alrasheed AA, Alduraywish AA, Alreshidi FS, et al. The prevalence of hypercholesterolemia and associated risk 
factors in Al-Kharj population, Saudi Arabia: a cross-sectional survey. BMC Cardiovasc Disord. 2021;21:22.

3. Durrington P. Dyslipidaemia. Lancet. 2003;362:717-31.

4. Ullah S, Jassal S. Serum lipid profile as an etiology of vertigo: A study. J Evid Based Med Healthc. 2015;2:5747-53.

5. Rosa Cde O, Dos Santos CA, Leite JI, Caldas AP, Bressan J. Impact of nutrients and food components on dyslipidemias: what is the evidence? Adv Nutr. 2015;6:70311.

6. Gadelha C, Bezerra AN. Effects of probiotics on the lipid profile: systematic review. J Vasc Bras. 2019;18:e20180124.

7. Anandharaj M, Sivasankari B, Parveen Rani R. Effects of Probiotics, Prebiotics and Synbiotics on Hypercholesterolemia: A Review. Chinese Journal of Biology. 2014;2014:1-7.
8. Kumar M, Nagpal R, Kumar R, Hemalatha R, Verma V, Kumar A, et al. Cholesterollowering probiotics as potential biotherapeutics for metabolic diseases. Exp Diabetes Res. 2012;2012:902917.

9. Mo R, Zhang X, Yang Y. Effect of probiotics on lipid profiles in hypercholesterolaemic adults: A metaanalysis of randomized controlled trials. Med Clin. 2019;152:473-81.

10. Lye HS, Rusul G, Liong MT. Removal of cholesterol by lactobacilli via incorporation and conversion to coprostanol. J Dairy Sci. 2010;93:1383-92.

11. Tomaro-Duchesneau C, Jones ML, Shah D, Jain P, Saha S, Prakash S. Cholesterol assimilation by Lactobacillus probiotic bacteria: an in vitro investigation. Biomed Res Int. 2014;2014:380316. 
Table 1. Literature searching strategy

\begin{tabular}{|c|c|c|}
\hline Database & Search Strategy & Hits \\
\hline Pubmed & $\begin{array}{l}(((\text { hypercholesterol*[Title/Abstract]) AND (probiotic[MeSH Terms])) OR } \\
\text { (probiotics[MeSH Terms])) AND (lipid profil*[Title/Abstract]) }\end{array}$ & 151 \\
\hline $\begin{array}{l}\text { Cochrane } \\
\text { Library }\end{array}$ & $\begin{array}{ll}\# 1 & \text { MeSH descriptor: [Hypercholesterolemia] explode all trees, } \\
& \mathrm{N}=3503 \\
\# 2 & \text { MeSH descriptor: [Probiotics] explode all trees, } \mathrm{N}=2134 \\
\# 3 & \text { lipid profil } *, \mathrm{~N}=13209 \\
\# 4 & \# 1 \text { AND \#2 AND \#3, } \mathrm{N}=5\end{array}$ & 5 \\
\hline Scopus & 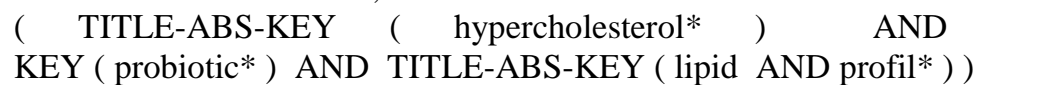 & TITLE-ABS- \\
\hline
\end{tabular}

\section{Table 2. Study characteristics}

\begin{tabular}{ll}
\hline \multicolumn{1}{c}{ Characteristics } & \multicolumn{1}{c}{ Mo R, et al. ${ }^{\mathbf{9}}(\mathbf{2 0 1 9})$} \\
\hline Prediction & Probiotics have efficacy in lowering serum lipid concentrations \\
Patient & Hypercholesterolemic adults \\
Determinant & Intervention: probiotics supplementation \\
& Comparison: placebo or no intervention \\
Study design & Meta-analysis of randomized controlled trials \\
Outcome & Probiotics supplementation significantly decreased TC level $-0.25 \mathrm{mmol} / \mathrm{L}(95 \% \mathrm{CI}$ : \\
& $-0.39--0.12 ; \mathrm{p}=0.0002)$, heterogeneity $\left(I^{2}=85 \%, \mathrm{p}<0.01\right)$ and $\mathrm{LDL}-\mathrm{C}$ level -0.17 \\
& mmol/L $(95 \% \mathrm{CI}:-0.25--0.09 ; \mathrm{p}<0.0001)$, heterogeneity $\left(I^{2}=67 \%, \mathrm{p}<0.01\right)$ \\
& Probiotics supplementation decreased HDL-C level $-0.02 \mathrm{mmol} / \mathrm{L}(95 \% \mathrm{CI}-0.05-$ \\
& $0.02 ; \mathrm{p}=0.37)$, heterogeneity $\left(I^{2}=80 \%, \mathrm{p}<0.01\right)$, but not statistically significant \\
& Probiotics supplementation changes TG level 0.01 mmol/L $(95 \% \mathrm{CI}:-0.02-0.05 ; \mathrm{p}$ \\
& $=0.51)$, homogeneity $\left(I^{2}=0 \%, \mathrm{p}=0.71\right)$, but not statistically significant \\
\hline
\end{tabular}

Abbreviations:

HDL-C: high-density lipoprotein cholesterol; LDL-C: low-density lipoprotein cholesterol; TC: total cholesterol; TG: triglycerides

Table 3. Relevance criteria

\begin{tabular}{|c|c|c|c|}
\hline & Similarity population & Similarity determinant & Similarity outcome \\
\hline Mo R. et al. ${ }^{9}(2019)$ & + & + & + \\
\hline
\end{tabular}

\section{Table 4. Validity criteria}

\begin{tabular}{|c|c|c|c|c|c|c|c|c|c|}
\hline Articles & 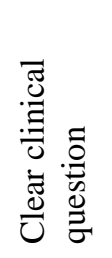 & 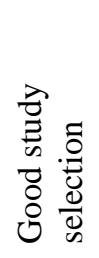 & 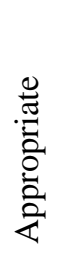 & 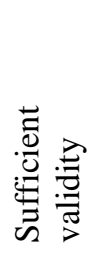 & 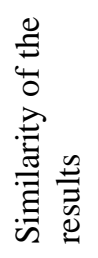 & 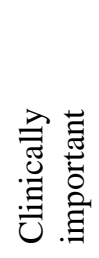 & 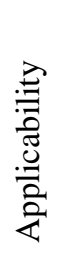 & Recommendation & $\begin{array}{l}\text { Level of } \\
\text { Evidence }\end{array}$ \\
\hline $\begin{array}{c}\text { Mo R. et } \\
\text { al. }^{9} \\
(2019)\end{array}$ & + & + & + & + & - & + & + & A & 1a- \\
\hline
\end{tabular}

Note:

1a -: Systematic Review with heterogeneity 
Table 5. Critical appraisal of systematic review

Critical appraisal questions

Mo R, et al. ${ }^{9}$ (2019)

\begin{tabular}{|c|c|c|}
\hline$\stackrel{\tilde{0}}{\stackrel{0}{0}}$ & What question (PICO) did the systematic review address? & $\begin{array}{l}\text { P: Hypercholesterolemic adults } \\
\text { I: Probiotics supplementation } \\
\text { C: Placebo or no treatment } \\
\text { O: Lipid profile }\end{array}$ \\
\hline$\stackrel{000}{:}$ & Is it unlikely that important, relevant studies were missed? & Yes \\
\hline 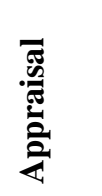 & $\begin{array}{l}\text { Were the criteria used to select articles for inclusion } \\
\text { appropriate? }\end{array}$ & Yes \\
\hline 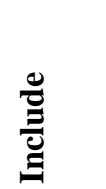 & $\begin{array}{l}\text { Were the included studies sufficiently valid for the type of } \\
\text { question asked? }\end{array}$ & Yes \\
\hline$\frac{\hat{\Xi}}{\frac{\tilde{\sigma}}{0}}$ & Were the results similar from study to study? & No \\
\hline 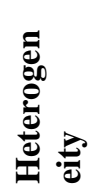 & What were the results? & Yes \\
\hline
\end{tabular}




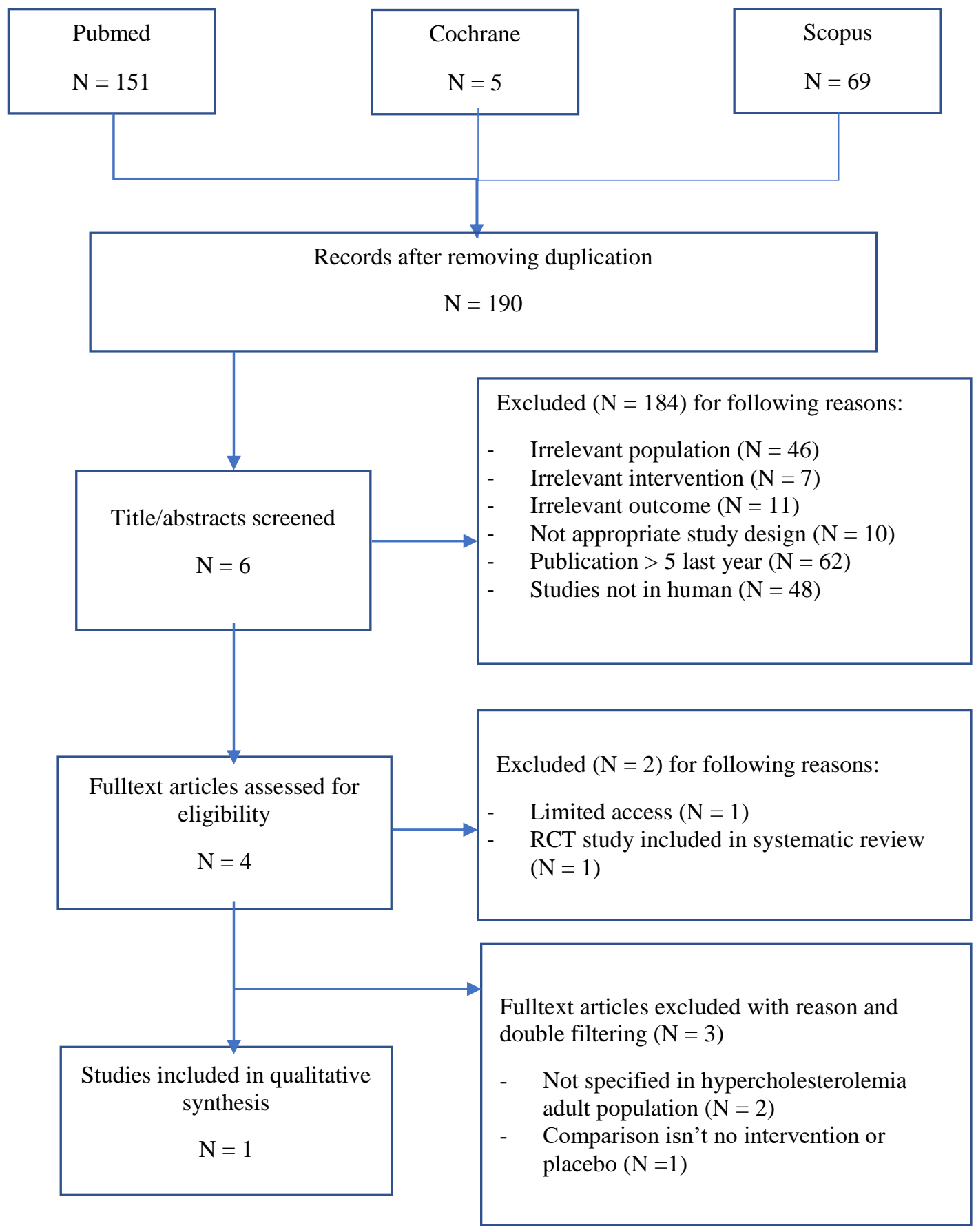

Figure 1. Prisma flow diagram on literature searching 